\title{
Clinically Indeterminate Breast Lesions with Normal Imaging: A Retrospective Study in a Symptomatic Breast Care Unit
}

\author{
Emre Pakdemirli (iD) ${ }^{1,}$, Mohamed Elkorety (iD) ${ }^{2}$ and Sherif Monib (iD) ${ }^{2}$ \\ ${ }^{1}$ Breast Care Unit, Department of Radiology, West Hertfordshire Hospitals NHS Trust, St. Albans City Hospital, St. Albans, United Kingdom \\ ${ }^{2}$ St. Albans Breast Care Unit, West Hertfordshire Hospitals NHS Trust, St. Albans, United Kingdom \\ "Corresponding author: Breast Care Unit, West Hertfordshire Hospitals NHS Trust, St. Albans City Hospital, St. Albans, United Kingdom. Email: dremrep@yahoo.co.uk
}

Received 2020 April 21; Revised 2020 August 12; Accepted 2020 August 16.

\begin{abstract}
Background: Triple assessment of breast pathologies is a very important pathway to detect breast cancers earlier.

Objectives: To ascertain the necessity of clinical-guided core biopsy (CGCB) or fine-needle aspiration cytology (FNAC) for investigating clinically indeterminate breast lesions with no significant imaging findings.

Patients and Methods: Retrospective analysis of 72 patients who had clinical core biopsy or fine-needle cytology was carried out to investigate clinically indeterminate breast lesions with normal imaging during the period from September 2017 to September 2019. Results: Out of 72 patients, 61 clinically indeterminate breast lesions (P3) were investigated and showed that 39 lesions (63.9\%) were graded as B1, 17 lesions (27.8\%) were graded as B2, two lesions (3.2\%) were graded as B3 showing atypia, no lesions were graded as B4, two lesions (3.2\%) were graded as B5 (one [1.6\%] was found to be invasive lobular carcinoma [ILC] and the other one [1.6\%] was found to be metastatic colorectal cancer to the breast), while one lesion investigated by FNAC was graded as C2 (1.6\%).

Conclusion: CGCB or FNAC is still necessary and vital for investigating clinically indeterminate breast lesions with normal imaging.
\end{abstract}

Keywords: Indeterminate Breast Lesions, Ultrasound Scan, Mammogram, Core Biopsy, Fine-Needle Aspiration Cytology

\section{Background}

Breast cancer is one of the top causes of cancer related mortalities among females. It was responsible for 2.1 million new cases and 626,679 cancer related deaths that were reported worldwide in 2018. Fair access to clinical and radiological assessment is crucial to early detection of breast cancer (1).

Although some guidelines and task forces have discarded clinical breast examination (CBE) (2), there is no doubt that CBE performed by appropriately trained breast physicians plays an important role in detecting breast cancer (3). Triple assessment ([CBE], imaging, and histopathological assessment) of breast pathologies is a very important method to detect breast cancer earlier.

The vast majority of palpable lumps are benign, but one cannot give \%100 reassurance in terms of excluding malignant masses (4). Roughly, one-third of breast biopsies come back with diagnosis of breast cancer (5). This retrospective study aimed to ascertain the necessity of clinical-guided core biopsy (CGCB) or fine needle aspira- tion cytology (FNAC) for investigating clinically indeterminate breast lesions that have no significant imaging findings.

\section{Objectives}

The objective was to ascertain the necessity of clinicalguided core biopsy or fine-needle aspiration cytology for investigating clinically indeterminate breast lesions with no significant imaging findings.

\section{Patients and Methods}

We conducted a retrospective observational analysis of our current practice of 72 patients (who had undergone clinical core biopsy or FNAC) to investigate clinically palpable breast lesions that had normal imaging during the period from September 2017 to September 2019.

Based on our inclusion and exclusion criteria, we excluded 16 patients who were clinically graded as P4/P5, so the final number of patients included was 56 patients. 
Clinical and radiological assessment pathway for breast care unit patients is demonstrated in Figure 1. Radiological examinations were carried out by four dedicated breast radiologists who are board certified from the United Kingdom (UK) (the first radiologist with more than 30 years of experience, the second and third with more than 15 years of experience, and the fourth, with two years of experience).

Ultrasound scans were carried out with the patient in the supine position, using two GE Logiq E9 stand alone machines, with transverse probes (ML6-15 MHz transducer) and elastography, color and power Doppler options.

Mammographic examinations were carried out in the standing position using GE Senographe Essential ${ }^{\mathrm{TM}}$ digital mammography, and average breast thickness ( $60 \mathrm{~mm}$, mAs 86.4, Kv 31, filter Rh [Rhadium], Target Rh).

Clinical core biopsies were performed by dedicated UK board certified breast surgeons, breast physicians and breast care nurses using 14 G AchieveTM core biopsy needle fully cocked technique.

Data was collected from the hospital clinic biopsy register, info-flex (Electronic patient records), and imaging and histopathology reports.

Clinical breast examination (CBE) and mammographic and ultrasound grading used for reporting was based on the UK 5-point breast imaging and reporting data system (UK BI-RADS) (R1: normal, R 2: benign 3: indeterminate /probably benign, R4: suspicious of malignancy and R5:highly suspicious of malignancy), which matched the 5-point clinical P grading system (U1, normal; U2, benign; $\mathrm{U} 3$, indeterminate/probably benign; U4, suspicious of malignancy; and U5, highly suspicious of malignancy), which matched the 5-point clinical P grading system (Table 1) (6, 7).

None of the 56 patients had post biopsy complications and all underwent written informed consent prior to the biopsy procedures.

\section{Results}

The total number of patients was 56 patients, the median age and mean age (standard deviation [SD]) were 46 years, and 48.3 years (14.8), respectively. Ten patients (17.8\%) were younger than 40 years, while 46 patients ( $82.1 \%$ ) were older than 40 years.

Fifty-five patients (98.2\%) were female and only one patient (1.7\%) was male.
The total number of examined breast lesions was 61 lesions, 27 patients (48.2\%) had lesions on the right side, and 24 (42.8\%) had left-sided lesions, while five (8.9\%) had bilateral breast lesions.

Clinical assessment of index breast lesions was graded using P grading. All 61 included lesions were graded as P3.

Imaging modalities in the one-stop clinic included breast ultrasound as well as mammographic assessment, ultrasound scan was graded as U1 in 47 lesions (77\%), U2 in 13 lesions (21.3\%), and none were graded as U3, U4, and U5. One lesion (1.6\%) did not have an ultrasound scan, as there was no suspicious lesion seen on mammogram.

Mammograms were not carried out for 20 patients (one male patient (1.7\%), 10 patients (17.8\%) younger than 40 years, and nine patients (16\%) who had recent mammograms), 32 lesions (52.4\%) were graded as R1 and nine lesions (14.7\%) were graded as R2, none were graded as R3, R4, or R5.

Histological assessment of clinical core biopsy revealed that 39 lesions (63.9\%) were graded as B1, of which 17 were predominantly adipose tissue, and 22 were fibroglandular breast tissue.

Seventeen lesions (27.8\%) were graded as B2, of which 10 were fibrous breast tissue, two fibrocystic disease, two chronic inflammation, one fat necrosis, one columnar cell change, and one pseudoangiomatous stromal hyperplasia (PASH).

Two lesions (3.2\%) were graded as B3, one was atypical intraductal epithelial.

proliferation (AIDEP), and the other was flat epithelial atypia.

Two lesions (3.2\%) were graded as B5 (one [1.6\%] was found to be invasive lobular carcinoma [ILC], and the other one [1.6\%] was found to be metastatic colorectal cancer to the breast). One lesion (1.6\%) was investigated by FNAC; cytology was graded as C2 showing adipocytes in keeping with a lipoma (Figure 2 ).

\section{Discussion}

Breast cancer is the most common cancer in the UK, accounting for $15 \%$ of all newly diagnosed cancer cases per year. Fifty-five thousand women are diagnosed with breast cancer in the UK every year, (150 diagnosed every day).

The incidence of breast cancer is estimated to rise by $2 \%$ in the UK between 2014 and 2035. It may well reach to 210 cases per 100,000 females by 2035 (8). While a palpable breast lump is the most common presenting symptom 


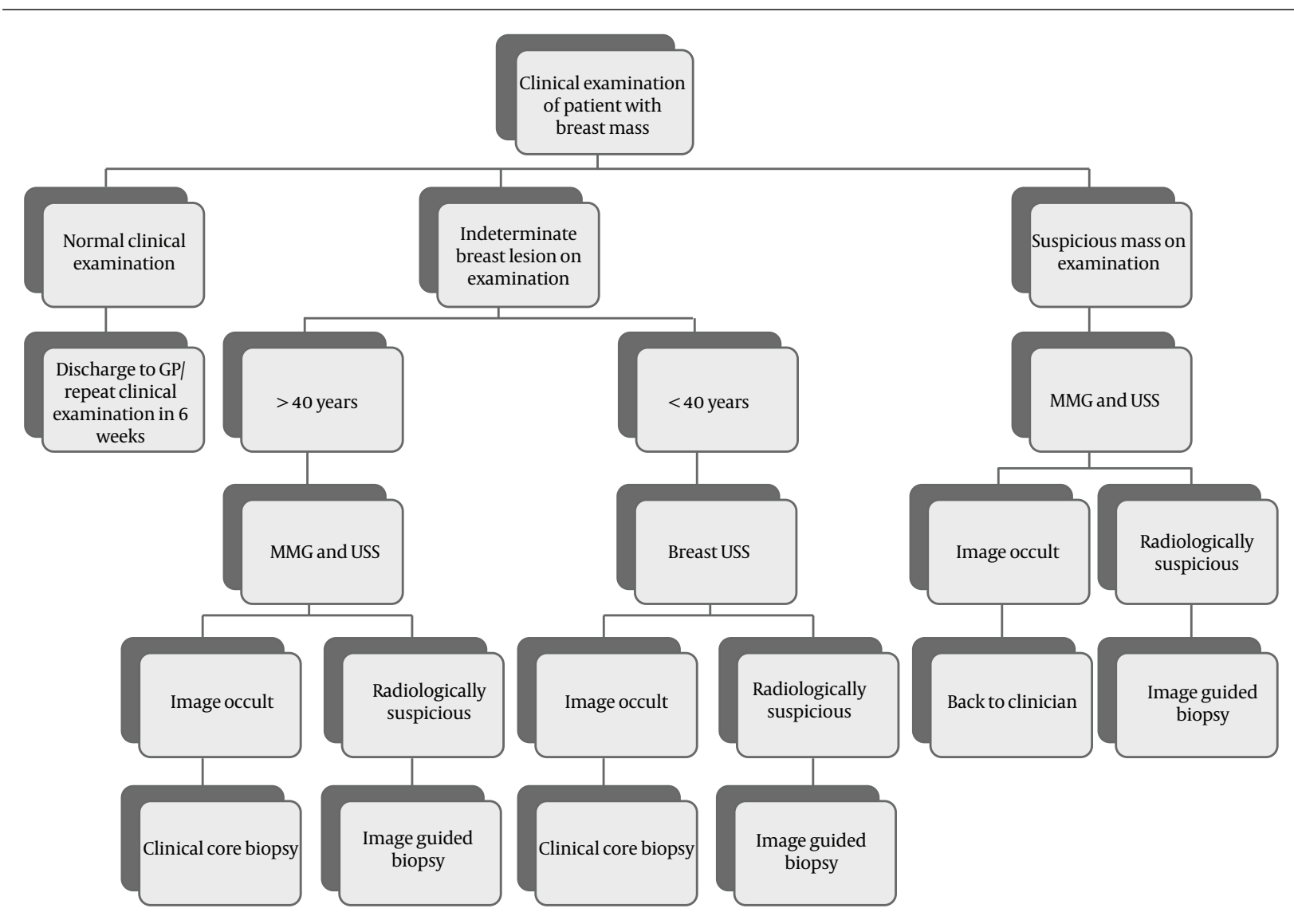

\begin{tabular}{|c|c|c|c|c|c|}
\hline Clinical & Ultrasound scan & Mammogram & MRI & Histopathology & Cytology \\
\hline \multicolumn{6}{|l|}{ Px: Previous biopsy } \\
\hline P1: Normal & U1: normal & R1: normal & MRI 1: normal & B1: normal & C1: normal \\
\hline P2: Benign & U2: benign & R2: benign & MRI2: benign & B2: benign & C2: benign \\
\hline P3: Indeterminate & U3: indeterminate & R3: indeterminate & MRI3: indeterminate & B3: indeterminate & C3: indeterminate \\
\hline P4: Suspicious & U4: suspicious & R4: suspicious & MRI4: suspicious & B4: suspicious & C4: suspicious \\
\hline \multirow{2}{*}{ P5: Highly suspicious } & \multirow{2}{*}{ U5: malignant } & \multirow{2}{*}{ R5: malignant } & \multirow{2}{*}{ MRI5: malignant } & $\begin{array}{c}\text { B5a: non invasive } \\
\text { cancer }\end{array}$ & \multirow{2}{*}{ C5: malignant } \\
\hline & & & & B5b: invasive cancer & \\
\hline
\end{tabular}

Abbreviation: MRI, magnetic resonance imaging.

${ }^{\mathrm{a}}$ Source: $(6,7)$.

of breast cancer, as reported by Mutar et al. (9) representing $71.3 \%$, breast pain represents $18.9 \%$ of presenting symptoms.

Clinical breast examination usually precedes imaging for patients presenting to the breast clinic. Mutar et al.
(9) and Koo et al. (10) found that patients usually demand breast examination if they experience a persistent lump other than other kinds of breast complaints.

Clinical breast examination is a simple non-invasive and cheap way to detect breast cancers, but adjunct imag- 


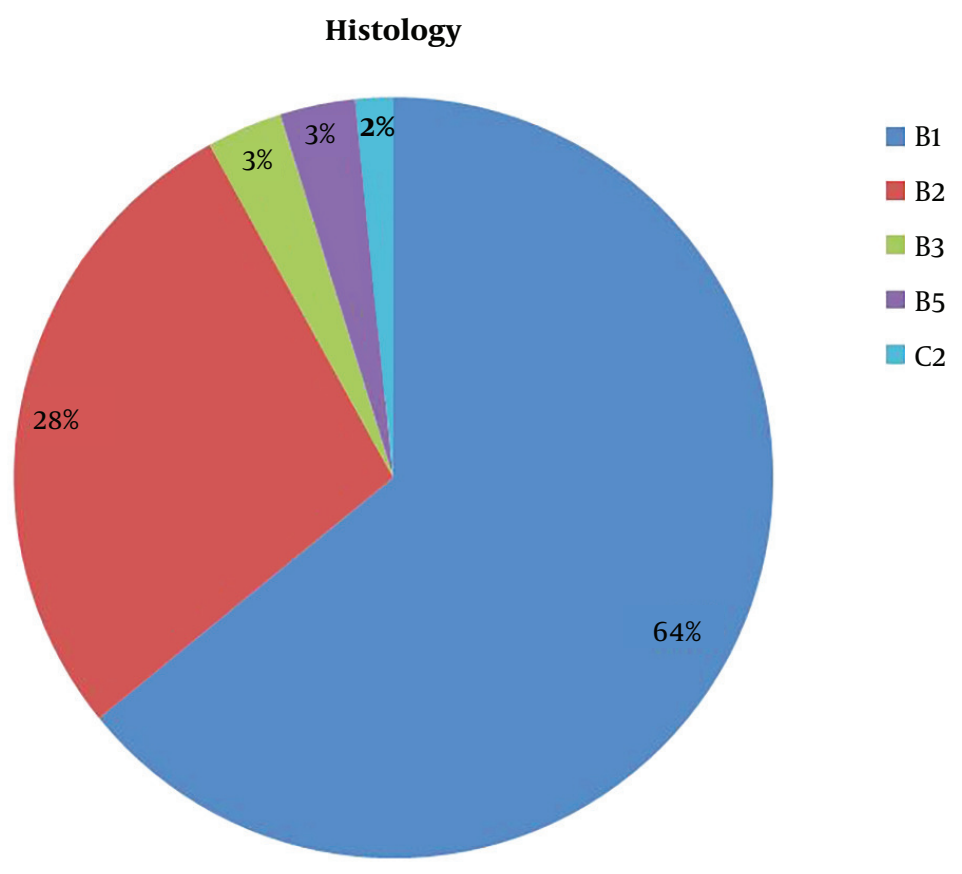

Figure 2. Histopathological distribution of indeterminate breast lesions

ing should be added to increase the final accuracy.

$60 \%-80 \%$ of breast biopsies usually show benign findings (11-13). Breast ultrasound and mammography could increase breast cancer detection rate by 4.2 cancers per 1000 screened women compared to women who undergo screening mammography alone (14).

Benson et al. reported the sensitivity of breast ultrasound as $89 \%$ (15). Houssami et al. (16) reported that the overall sensitivity of mammography and ultrasound is $96 \%$.

The perception of a subtle mass or cluster of microcalcifications can be picked up by mammograms in case the image contrast is adjusted. Pisano et al. (17) and Yunus et al. (18) reported about $25 \%$ - $43 \%$ of non-palpable cancers can be detected on mammography in the presence of microcalcification.

In addition, Pisano et al. (19) found that the sensitivity and specificity of digital mammography in pre- or perimenopausal women less than 50 years with non-dense breasts is $85 \%$ and $90 \%$, respectively.

According to a study conducted by Dennis et al. (20), they followed up 540 breast lumps without ultrasound findings for at least 2 years, and they found that none of the lumps turned to be breast cancer or any other form of malignancy. This interesting result represents a negative predictive value of ultrasound is reaching 100\%. Therefore, they advised ignoring clinical breast biopsy when imaging is negative (20).

According to Kaiser et al. (21), who conducted a prospective study with a patient cohort of 103 , they followed up palpable breast thickening patients and reached a 100\% negative predictive value of ultrasound imaging. Houssami et al. (22) reported that clinically guided biopsy (CGB) could have a high false negative rate compared to image-guided core or FNA sampling. Ward et al. (23) found that the diagnostic accuracy increases in case of image guided sampling.

Larger patient cohort studies have already demonstrated that core biopsy sampling approach has a better diagnostic outcome to FNAB in terms of sensitivity, specificity and correct histological grading (24-26).

Gumus et al. (27) advised that breast ultrasound scan should be performed for clinically palpable, mammographically occult breast lesions followed by clinically or ultrasound guided core biopsy.

The vast majority of our patients had a normal or benign findings on mammogram and ultrasound scans (Figures 3 and 4), yet clinicians opted to do a clinically guided needle biopsy to ascertain the final diagnosis.

In our patient cohort, we picked up two malignancies 

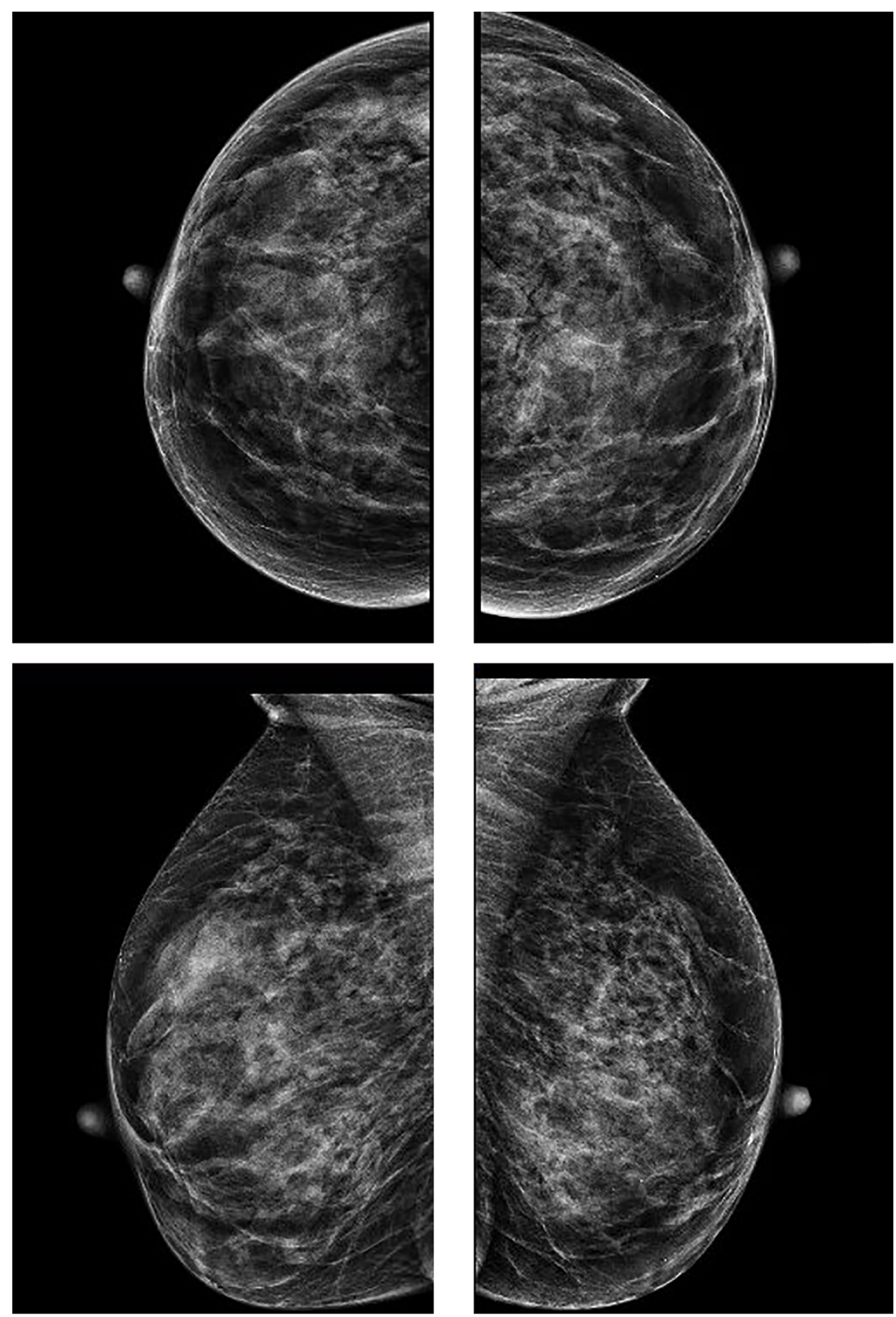

Figure 3. Bilateral mammogram of a 93-year-old patient presenting with palpable clinically indeterminate breast lump showing no suspicious findings 

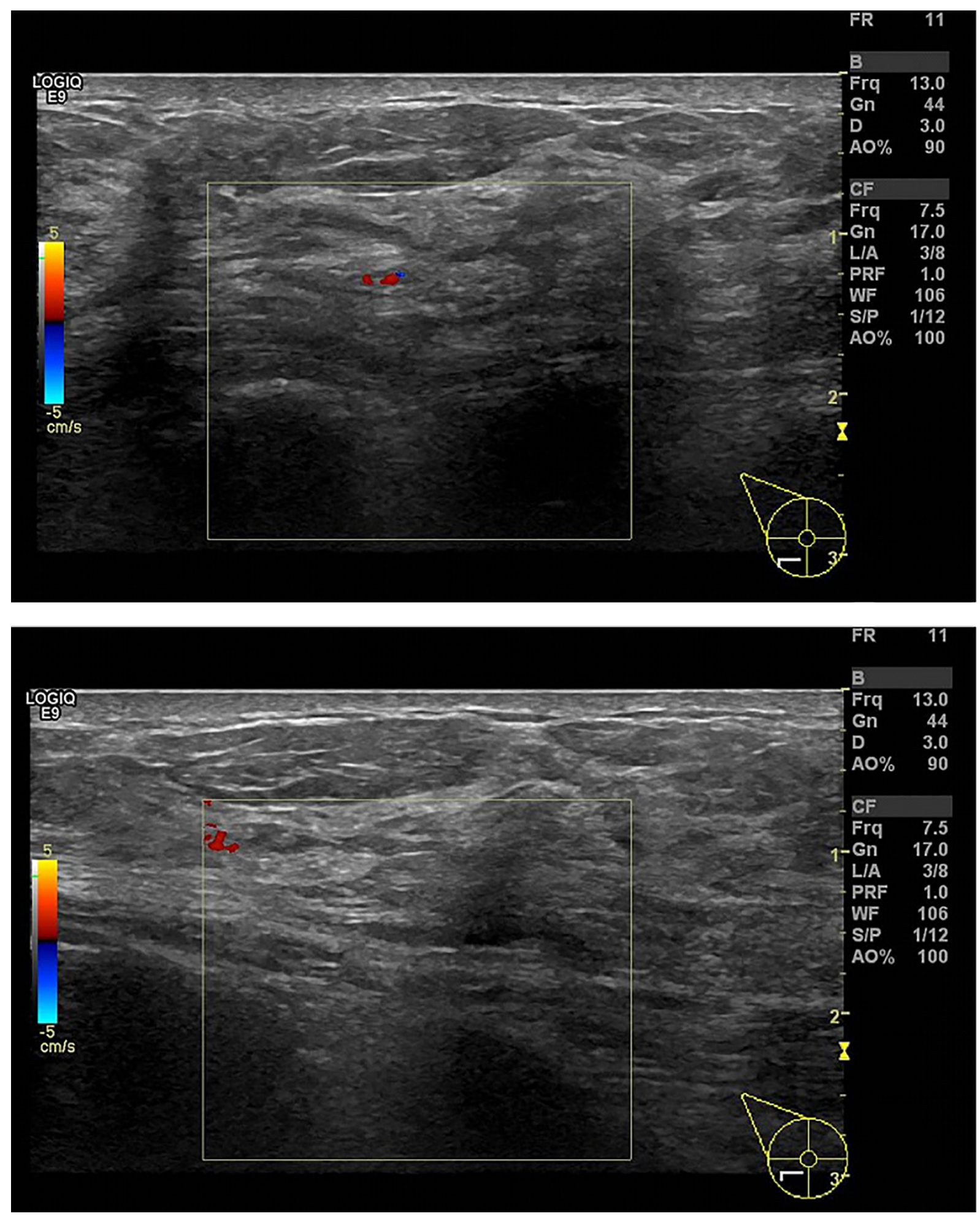

Figure 4. Two-breast B-mode ultrasound scan pictures with Doppler facility of the same patient showing non-specific shadowing in the palpable area with non-significant Doppler signals 
while Dennis et al. (20) and Kaiser et al. (21) reported none. One case was found to be ILC which is well known to be a hideous malignancy and can disguise itself even on MRI, while the second case was metastatic colorectal cancer to the breast. Therefore, any indeterminate breast lesions with negative imaging should be biopsied clinically, preferably with a core biopsy. The value of breast MRI scan can be arguable, it could be very valuable for most breast cancers, but hideous ILC or even invasive ductal carcinoma (IDC) can still be MRI negative (28).

Clinical assessment of palpable breast masses with normal imaging is challenging and harbour potential breast cancer misses. A no biopsy approach policy would result in potential delay in diagnosis. Ignoring the normal imaging findings and doing relentless CGB for all breast patients may result in too many unnecessary procedures.

Salzman et al. (29) introduced an algorithm/pathway for the management of patients presenting with breast lumps advising when to proceed with clinical guided biopsy and when to refer for imaging. In our breast unit, we have been using a similar algorithm with differences to meet our imaging guidelines (Figure 1).

Limitations of our study included, a small sample size, symptomatic patient cohort without screening patients, and clinicians' and radiologists wide range of experience.

In conclusion, CGCB or FNAC are still valid approaches for investigating clinically indeterminate breast lesions with normal imaging. Multi-centric studies including a larger cohort of patients are still needed to come up with more robust results to deliver better patient care.

\section{Footnotes}

Authors' Contributions: EP developed the original idea and the protocol, abstracted and analyzed data, wrote the manuscript, and was a guarantor. ME and SM contributed to the development of the protocol, abstracted data, and prepared the manuscript.

Conflict of Interests: The authors declare that there are no conflicts of interest.

Ethical Approval: No ethical approval was needed for this study, as it was a retrospective analysis.

Funding/Support: This research received no specific grant from any funding agency in the public, commercial, or not-for-profit sectors.

\section{References}

1. Bray F, Ferlay J, Soerjomataram I, Siegel RL, Torre LA, Jemal A. Global cancer statistics 2018: GLOBOCAN estimates of incidence and mortality worldwide for 36 cancers in 185 countries. CA Cancer J Clin. 2018;68(6):394-424. doi: 10.3322/caac.21492. [PubMed: 30207593].

2. Nelson HD, Tyne K, Naik A, Bougatsos C, Chan BK, Humphrey L, et al. Screening for breast cancer: an update for the U.S. Preventive Services Task Force. Ann Intern Med. 2009;151(10):727-37. W237-42. doi: 10.7326/0003-4819-151-10-200911170-00009. [PubMed: 19920273]. [PubMed Central: PMC2972726].

3. Provencher L, Hogue JC, Desbiens C, Poirier B, Poirier E, Boudreau D, et al. Is clinical breast examination important for breast cancer detection? Curr Oncol. 2016;23(4):e332-9. doi: 10.3747/co.23.2881. [PubMed: 27536182]. [PubMed Central: PMC4974039].

4. Ma I, Dueck A, Gray R, Wasif N, Giurescu M, Lorans R, et al. Clinical and self breast examination remain important in the era of modern screening. Ann Surg Oncol. 2012;19(5):1484-90. doi: 10.1245/s10434-0112162-9. [PubMed: 22160521].

5. Taylor KJ, Merritt C, Piccoli C, Schmidt R, Rouse G, Fornage B, et al. Ultrasound as a complement to mammography and breast examination to characterize breast masses. Ultrasound Med Biol. 2002;28(1):1926. doi: 10.1016/s0301-5629(01)00491-4. [PubMed: 11879948].

6. Taylor K, Britton P, O'Keeffe S, Wallis MG. Quantification of the UK 5-point breast imaging classification and mapping to BI-RADS to facilitate comparison with international literature. $\mathrm{Br} J \mathrm{Ra}$ diol. 2011;84(1007):1005-10. doi: 10.1259/bjr/48490964. [PubMed: 22011830]. [PubMed Central: PMC3473699].

7. [No author listed]. NHS Cancer Screening Programmes and the Royal College of Pathologists Pathology reporting of breast disease. London: NHSBSP; 2001.

8. Cancer Research UK. Breast cancer statistics. 2019, [cited 11 Nov 2019]. Available from: https://www.cancerresearchuk.org/healthprofessional/cancer-statistics/statistics-by-cancer-type/breastcancer.

9. Mutar MT, Goyani MS, Had AM, Mahmood AS. Pattern of Presentation of Patients With Breast Cancer in Iraq in 2018: A Cross-Sectional Study. J Glob Oncol. 2019;5:1-6. doi: 10.1200/JGO.19.00041. [PubMed: 31721627]. [PubMed Central: PMC6882514].

10. Koo MM, von Wagner C, Abel GA, McPhail S, Rubin GP, Lyratzopoulos G. Typical and atypical presenting symptoms of breast cancer and their associations with diagnostic intervals: Evidence from a national audit of cancer diagnosis. Cancer Epidemiol. 2017;48:140-6. doi: 10.1016/j.canep.2017.04.010. [PubMed: 28549339]. [PubMed Central: PMC5482318].

11. Hampton T. Breast cancer symposium highlights risk, recurrence, and research trials. JAMA. 2012;307(4):348-50. doi: 10.1001/jama.2012.30. [PubMed: 22274677].

12. Ravi C, Rodrigues G. Accuracy of clinical examination of breast lumps in detecting malignancy: a retrospective study. Indian J Surg Oncol. 2012;3(2):154-7. doi: 10.1007/s13193-012-0151-5. [PubMed: 23730103]. [PubMed Central: PMC3392478].

13. Bassett LW, Liu TH, Giuliano AE, Gold RH. The prevalence of carcinoma in palpable vs impalpable, mammographically detected lesions. AJR Am J Roentgenol. 1991;157(1):21-4. doi: 10.2214/ajr.157.1.1646562. [PubMed: 1646562].

14. Berg WA, Blume JD, Cormack JB, Mendelson EB, Lehrer D, Bohm-Velez $\mathrm{M}$, et al. Combined screening with ultrasound and mammography vs mammography alone in women at elevated risk of breast cancer. JAMA. 2008;299(18):2151-63. doi: 10.1001/jama.299.18.2151. [PubMed: 18477782]. [PubMed Central: PMC2718688]. 
15. Benson SR, Blue J, Judd K, Harman JE. Ultrasound is now better than mammography for the detection of invasive breast cancer. Am J Surg. 2004;188(4):381-5. doi:10.1016/j.amjsurg.2004.06.032. [PubMed: 15474430].

16. Houssami N, Irwig L, Simpson JM, McKessar M, Blome S, Noakes J. Sydney Breast Imaging Accuracy Study: Comparative sensitivity and specificity of mammography and sonography in young women with symptoms. AJR Am J Roentgenol. 2003;180(4):935-40. doi: 10.2214/ajr.180.4.1800935. [PubMed: 12646432].

17. Pisano ED, Cole EB, Major S, Zong S, Hemminger BM, Muller KE, et al. Radiologists' preferences for digital mammographic display. The International Digital Mammography Development Group. Radiology. 2000;216(3):820-30. doi: 10.1148/radiology.216.3.ro0se48820. [PubMed: 10966717].

18. Yunus M, Ahmed N, Masroor I, Yaqoob J. Mammographic criteria for determining the diagnostic value of microcalcifications in the detection of early breast cancer.J Pakistan Med Assoc. 2004;54(1):24.

19. Pisano ED, Hendrick RE, Yaffe MJ, Baum JK, Acharyya S, Cormack JB, et al. Diagnostic accuracy of digital versus film mammography: exploratory analysis of selected population subgroups in DMIST. Radiology. 2008;246(2):376-83. doi: 10.1148/radiol.2461070200. [PubMed: 18227537]. [PubMed Central: PMC2659550].

20. Dennis MA, Parker SH, Klaus AJ, Stavros AT, Kaske TI, Clark SB. Breast biopsy avoidance: the value of normal mammograms and normal sonograms in the setting of a palpable lump. Radiology. 2001;219(1):186-91. doi: 10.1148/radiology.219.1.r01ap35186. [PubMed: 11274555].

21. Kaiser JS, Helvie MA, Blacklaw RL, Roubidoux MA. Palpable breast thickening: role of mammography and US in cancer detection. Radiology. 2002;223(3):839-44. doi: 10.1148/radiol.2233011166. [PubMed: 12034957].
22. Houssami N, Ciatto S, Ambrogetti D, Catarzi S, Risso G, Bonardi R, et al. Florence-Sydney Breast Biopsy Study: sensitivity of ultrasoundguided versus freehand fine needle biopsy of palpable breast cancer. Breast Cancer Res Treat. 2005;89(1):55-9. doi: 10.1007/s10549-004-14710. [PubMed: 15666197].

23. Ward ST, Shepherd JA, Khalil H. Freehand versus ultrasound-guided core biopsies of the breast: reducing the burden of repeat biopsies in patients presenting to the breast clinic. Breast. 2010;19(2):105-8. doi: 10.1016/j.breast.2009.12.003. [PubMed: 20074953].

24. Garg S, Mohan H, Bal A, Attri AK, Kochhar S. A comparative analysis of core needle biopsy and fine-needle aspiration cytology in the evaluation of palpable and mammographically detected suspicious breast lesions. Diagn Cytopathol. 2007;35(11):681-9. doi: 10.1002/dc.20721. [PubMed: 17924407].

25. Homesh NA, Issa MA, El-Sofiani HA. The diagnostic accuracy of fine needle aspiration cytology versus core needle biopsy for palpable breast lump(s). Saudi Med J. 2005;26(1):42-6. [PubMed: 15756351].

26. Pisano ED, Fajardo LL, Caudry DJ, Sneige N, Frable WJ, Berg WA, et al. Fine-needle aspiration biopsy of nonpalpable breast lesions in a multicenter clinical trial: results from the radiologic diagnostic oncology group V. Radiology. 2001;219(3):785-92. doi: 10.1148/radiology.219.3.ro1jn28785. [PubMed: 11376270].

27. Gumus H, Gumus M, Mills P, Fish D, Devalia H, Jones SE, et al. Clinically palpable breast abnormalities with normal imaging: is clinically guided biopsy still required? Clin Radiol. 2012;67(5):437-40. doi: 10.1016/j.crad.2011.10.010. [PubMed: 22119297].

28. Monib S, Pakdemirli E, Chong K. Mammographically and MRI occult breast cancer. BMJ Case Rep. 2019;12(6). doi: 10.1136/bcr-2019-230190. [PubMed: 31253664]. [PubMed Central: PMC6605947].

29. Salzman B, Collins E, Hersh L. Common breast problems. Am Fam Physician. 2019;99(8):505-514A. doi: 10.1258/1362180042721355. 